\title{
The Design of Drive Circuit for Light Source System of UV - LED Green Printing Dryer
}

\author{
Shao-Ru MA*, Jin-Yao LI, Gui-Shan ZHANG, Tao MI \\ Mechanical and Electrical Engineering Department, Beijing Institute of Graphic Communication \\ Beijing,Daxing,China \\ 1105399682@qq.com \\ ${ }^{*}$ Corresponding author
}

Keywords: UV-LED, curing system, drive circuit.

\begin{abstract}
UV-LED drying technology in printing is an energy-saving, environmentally friendly green drying technology. Compared with the traditional UV drying method, it can reduce $70 \%-90 \%$ of the power consumption; In addition, the drying device does not produce ozone, no VOC (volatile organic matter) emissions. At present, the technology has become a research hotspot in the printing area at home and abroad. Drive circuit of UV-LED light source system is one of the key technologies of UV-LED drying equipment, as a single UV-LED chip power is only a few watts, which can't meet the demands of drying power, it is necessary to form a number of chips that integrated UV-LED light source system, which requires that the drive circuit has high stability in order to improve the reliability and the service life of the drying device. In this paper, a light source system composed of 72 LED chips in array arrangement is introduced, and it mainly introduces that the driving circuit of the LED chip is directly driven by rectifying and valley fill circuit. The experiment and test results show that the driving circuit can not only meet the drying power requirements of LED light source system, but also compensate and improve the chip power according to the actual needs of the curing system, so that working reliability and service life of the drying device can be improved.
\end{abstract}

\section{Introduction}

Over the years, China's actively implemented the sustainable development strategies, which put energy conservation and emission reduction on an important strategic position. September 2010, the Ministry of Environmental Protection and the Press and Publication Administration jointly signed the Implementation of Green Printing Strategic Cooperation Agreement, marking the implementation of green printing to promote the formal launch[1]. October 8, 2011, the Press and Publication Administration and the Ministry of Environmental Protection jointly issued the Notice on the Implementation of Green Printing on China's implementation of green printing to make more comprehensive deployments and arrangements, the work of green printing implementation went into the A new phase. Green printing refers to the small impact on the ecological environment, less pollution, saving resources and energy printing, involving the whole process of printing production[2].

UV-LED printing and drying technology is an energy-saving, environmentally friendly green drying technology. Currently, most of the UV drying light source in the printing industry is still using the traditional high-pressure mercury lamp and metal halide lamp, such traditional UV lamp contains heavy metal pollutants, high energy consumption and short service life, needed frequent replacement. Compared with the traditional UV drying, UV-LED drying has the following advantages: 1. energy saving. It can significantly reduce energy consumption, reducing $80 \%-90 \%$ of the power consumption; 2.environmental protection. Drying device does not produce ozone, no VOC (volatile organic) emissions; 3.long service life. Can reduce the number of replacing lights. UV-LED lamp can serve 20,000 hours, while the traditional UV high-pressure mercury lamp can be only used for 1,000 hours; 4.high printing efficiency. UV-LED can be instantly turned on or off, without the need for UV lamp preheating and cooling time, printing efficiency has been greatly 
improved. 5. thermal radiation is very small. UV-LED is a cold light source, and heat radiation is very small, which reduces the heat generated during drying and will not stretch the printed materials heat deformation, particularly conducive to label printing and heat-sensitive printing material printing. At the same time, due to the small impact on the press, printing machinery will be impacted [3].

At present, there are some foreign enterprises that have carried the UV-LED drying equipment out earlier and become a mature product, such as Japan Ryobi (RYOBI), the United States Phoseon, the United States good music UV, the Germany's Heraeus, the United States Empire Screen Printing company, KBA, Roman and other companies [4]. At home, universities and enterprises to carry out researches in this area earlier are: Fu Dan University, Beijing Institute of Printing and other colleges and universities; Qingdao Hua Xun, Jiangsu Guang Fa Photoelectric, Shenzhen Ren, Shenzhen blue spectrum Rick and other companies [5].

If the key technology of UV-LED drying system can be overcome, the printing and packaging enterprises will be directly benefited, which can significantly reduce energy consumption, VOC emissions, and improve efficiency.

\section{The design of light source drive circuit of UV-LED drying device}

Structure of UV-LED Drying Device. UV-LED drying device consists of four parts, including the light source module, the light source system drive circuit, cooling module, single chip machine control module. As shown in Figure 1.

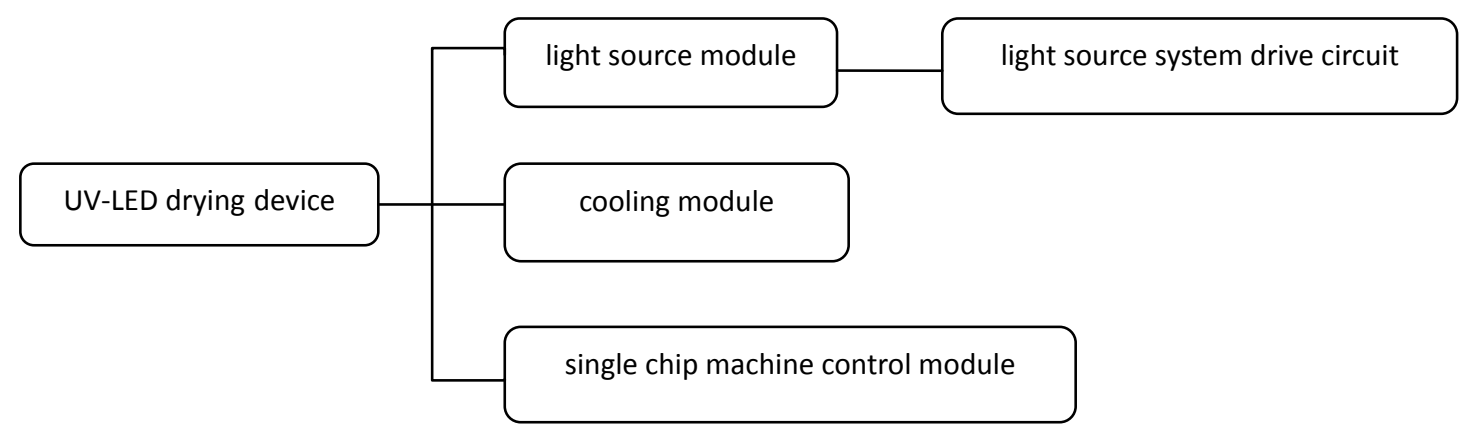

Fig. 1 UV-LED drying device block diagram

Design of Light Source Module Driving Circuit. In the UV-LED drying system, a single LED chip can't meet the requirement of power, it often requires multiple beads by series, parallel or mixed way to form a UV-LED light source system to achieve the actual production, the connection of UV-LED load directly related to its reliability and service life. In the design of light source system drive circuit, the general consideration is the cost and performance. When multiple LEDs are connected together, both the forward voltage and current must match so that multiple LED chips produce consistent brightness. The simplest way to achieve a constant current is to connect the LEDs in series with the forward voltage. The current numbers of LED chips connections are the following: 1. Series drive; 2.Parallel drive; 3.Hybrid drive. But there are disadvantages in these ways, This article chooses the way that each LED parallel Zener diode, which the Zener diode turn-on voltage than the LED's turn-on voltage is higher, so that it can ensure the remaining lamp beads can continue normal jobs, with the damage to lamp beads, As shown in Figure 2. 


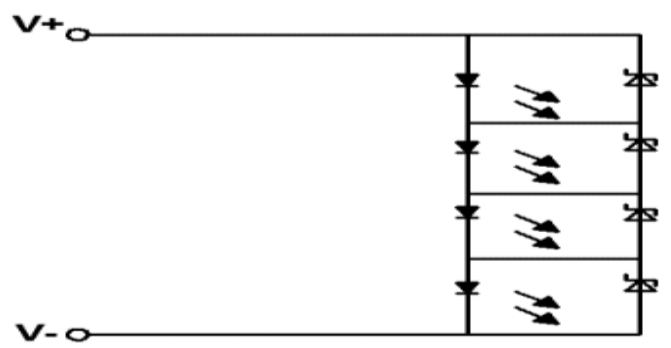

Fig. 2 LED Zener diode connected in parallel at both ends

The power supply of the LED chip is AC220V directly through the bridge rectification filter, the driving chip and the adaptive valley filling circuit and the light source module can be increased according to the power required of the actual work to improve the power of the UV-LED drying device. The light source drive circuit process diagram is shown in Figure 3.

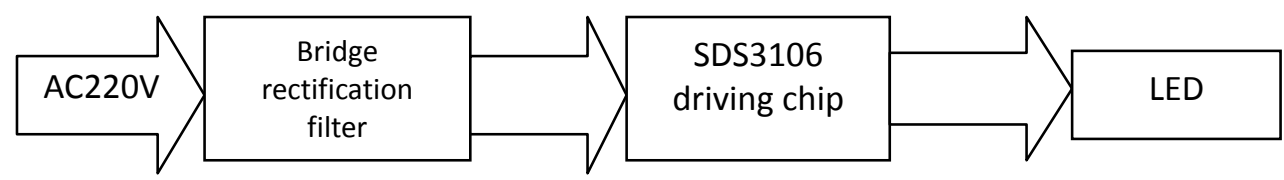

Fig. 3 LED drive circuit process diagram

Design of Rectifier Filter Circuit. In the design requirements of this chapter, the input voltage is AC220V, a single LED drive voltage is $3 \mathrm{~V}$, conduction current range is $0.1 \sim 700 \mathrm{~mA}$, LED drive circuit's main function is to convert AC voltage to DC voltage, and at the same time match the LED voltage and the current. Therefore, after AC220V power supply, through the rectifier filter network to achieve AC / DC conversion, as shown in Figure 4.Commonly the rectifier filter network used consists of diode and filter capacitors and other non-linear components, making that the drive power supply for the power supply presents non-linear impedance of the power system, in order to obtain a better filtering, usually select the larger capacitor value's filter capacitor.

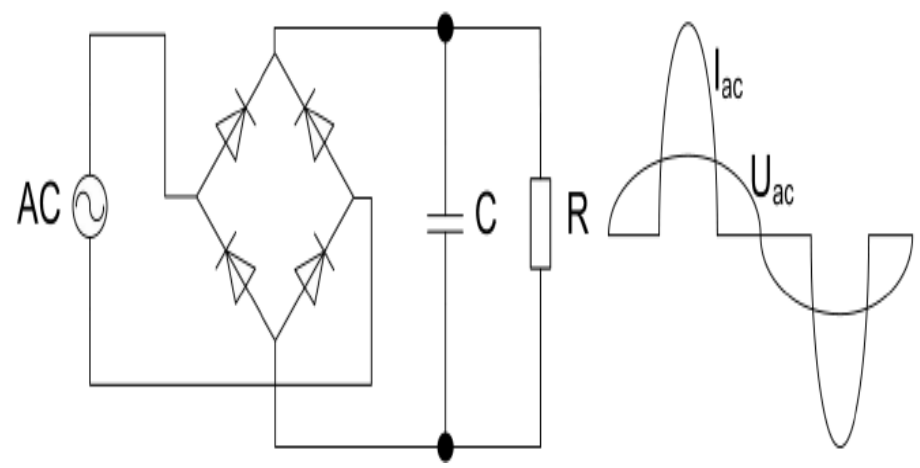

Fig. 4 Bridge rectifier circuit and voltage circuit waveform

Design of SDS 3106 Control Chip Circuit. Control chip of LED as the drive power is to detect the entire drive power supply circuit and control the overall operation of the integrated circuit, the choice of chip in the drive power of the design is of critical significance, all of the control circuit are connected around the chip design, the design of different chip circuit structure is not the same. In this design, the chip selection to take into account the function of the circuit, the cost, the structure, the simplification of structure, the stability and other factors, so that choose the SDS3106 chip.

SDS3106 is a single-channel high-voltage constant current LED driver chip. SDS3106 chip apply linear constant current technology, through external resistance to set the LED chip drive current, divided into two sections, flexible configuration, which can adapt to various LED 
application requirements from 3 to $18 \mathrm{~W}$, and conclude adaptive valley filling technology [6] to effectively improve the efficiency and linear adjustment of power system.

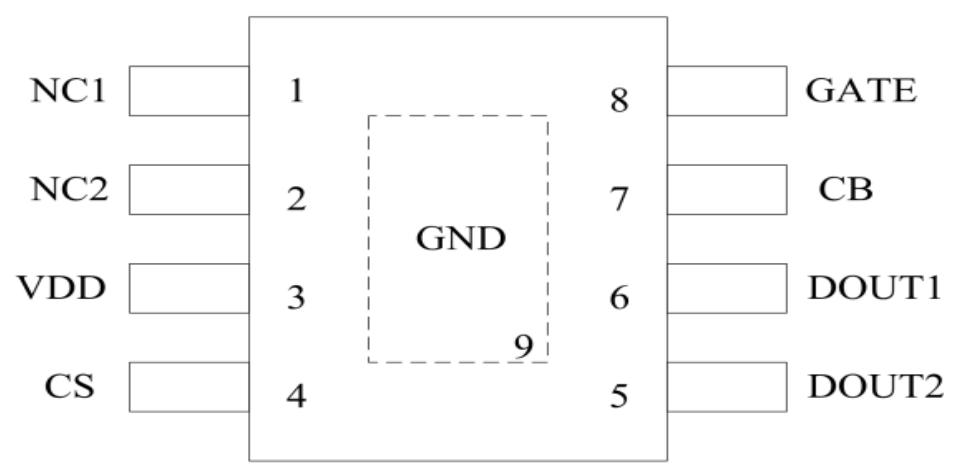

Fig. 5 Pin diagram of the SDS3106 chip

\section{Summary}

In this paper, the driving system of the light source system of the UV-LED green printing and drying device is studied. That the drive circuit of the light source system is composed of 72 pieces $395 \mathrm{~nm}$ LED chips arrayed is introduced. By connecting in series, and in parallel with a Zener diode on each chip. The technology of adaptive valley filling circuit and control chip is also introduced. The experimental results show that the adaptive valley filling technique has practical significance to the light source system driving circuit.

\section{References}

[1] Green Printing Technology Guide ${ }^{[\mathrm{M}]}$. Beijing: Printing Industry Press, (2011.9).

[2] Printing Environmental Protection Department of Environmental Development Center. Green

[3] Printing and China Environmental Label ${ }^{[\mathrm{M}]}$. Beijing: China Environmental Press, (2012.9).

[4] Lian Wenhua. UV curing technology of label printing industry ${ }^{[\mathrm{J}]}$. Printing Magazine,(2012.12).

[5] Daiichi Printing Co. Ltd. Two Presses with LED-UV in Full Operation Printing Applications on Both

[6] Paper and Film Developed ${ }^{[\mathrm{J}]}$.RYOBI LED-UV Printing System User Report. (2010).

[7] Lian Wenhua. UV curing technology in label printing industry ${ }^{[\mathrm{J}]}$.Printing Journal, (2012 .12).

[8] Zhou Zhimin, Zhou Jihai. Switch power supply power factor correction circuit design and application

[9] (first edition) ${ }^{[\mathrm{D}]}$. Beijing: People's Posts and Telecommunications Press. $(2004,11)$. 